\title{
AN IMPROVED EDGE DETECTION METHOD FOR IMAGE CORRUPTED BY GAUSSIAN NOISE
}

\author{
Xiao Wang ${ }^{1}$, Hui Xue ${ }^{1, *}$ \\ ${ }^{1}$ College of Information and Electrical Engineering, China Agricultural UniversityBeijing, \\ P.R. China, 100083 \\ * Corresponding author, Address: College of Information and Electrical Engineering, China \\ Agricultural University Beijing, P.R. China, 100083, Tel:86-13520508835, Email: \\ xue_huicn@yahoo.com.cn
}

Abstract: Due to the difficulty with extracting edge points and eliminating noise points from images, an improved maximizing objective function algorithm was proposed. More directions were added to relocate the edge points, at the same time, edge and noise characteristics were analyzed to separate and the noise points were eliminated by a proper threshold T. The comparison based on principle of the improved method, classical methods and the references methods is done, the simulation results indicated that the performance of the improved edge detection method was better than that of other compared algorithms.

Keywords: $\quad$ edge detection, Gaussian noise, directions, threshold

\section{INTRODUCTION}

Pattern recognition and machine vision were applied to extract the objects from the background in the images, as well in the agriculture field, contours of the objects can be used to recognize the plumpness of the plants. In order to extract the configuration parts from an image, the complete information of the image edge was necessary. Meanwhile, since the experimental images were always corrupted by Gaussian noise, the results of the edge detection were turned to be blurry or with some speckles.

\footnotetext{
Please use the following format when citing this chapter:

Wang, X. and Xue, H., 2009, in IFIP International Federation for Information Processing, Volume 294, Computer and Computing Technologies in Agriculture II, Volume 2, eds. D. Li, Z. Chunjiang, (Boston: Springer), pp. 1153-1159.
} 
In classical edge detection methods, such as Sobel Operator, LaplacianGaussian Operator, Roberts Operator, and Prewitt Operator etc, the maximums of the gradients were utilized to find image edge points (Duan et al., 2005). Because being sensitive to the noise, classical edge detection operators may not be practically used in the actual image processing. In order to discriminate edges with exact location, high signal-to-noise ratio and thin contours, lots of new edge detections algorithms are studied.

Zhao Chunjiang et al.(Zhao et al., 2005 ) proposed a new method based on the image gray-level characteristic, it made the process faster, but the location of the edge points in complex images was still a problem and some of the parameters needed to be settled by using plenty of experiments. Different masks were used to search the edge points: half neighborhood algorithm (Xu et al., 2006), more directions (Yao et al., 2007) etc., which could get better locations but noise always be preserved as edge points. Chung-Chia Kang and Wen-June Wang (Kang et al., 2007) proposed a method based on the maximizing objective function, which got the edges much better than the classical methods, but as well it was very sensitive to the noise. The proposed new edge detection method can be reasonably considered Gaussian noise reduction and accurate location of edge by adding more masks and separating edge and noise respectively.

\section{THE IMPROVED ALGORITHM}

The algorithm proposed in Kang et al., 2007 can get the edge points clearly because of its good discrimination capability, but when it combined with Gaussian noise, the image came to be blurry. Fig. 1 showed the 4 directions used in the original method.

Each direction mask was divided into two parts: $\mathrm{S}_{0}$ and $\mathrm{S}_{1}$, it was known that if the distances between $\mathrm{S}_{0}$ and $\mathrm{S}_{1}$ were large and the distances inside $\mathrm{S}_{0}$ and $S_{1}$ were small, then the edge intensity may be large. There was an objective function describing these distances were given as follows (Kang et al., 2007):

$$
f(i)=(L-1) \frac{N_{f}}{D_{f}}
$$

Where,

$$
N_{f}=\min \left(1, \frac{\left|m_{0}-m_{1}\right|}{\omega_{1}}\right)
$$




$$
D_{f}=1+\frac{1}{15} \sum_{\substack{p_{m}, p_{n} \in S_{1} \\ m>n, m \neq n}} \min \left(1, \frac{\left.\left|p_{m}-p_{n}\right|\right)}{\omega_{2}}\right)+\frac{1}{3} \sum_{\substack{p_{m}, p_{n} \in S_{1} \\ m>n, m \neq n}} \min \left(1, \frac{\left|p_{m}-p_{n}\right|}{\omega_{2}}\right)
$$

And $\omega_{1}=90, \omega_{2}=40$, and $m_{0}=(1 / 6) \sum_{p_{i} \in S_{0}} p_{i}, m_{1}=(1 / 3) \sum_{p_{i} \in S_{1}} p_{i}$

(Kang et al., 2007), $N_{f}$ describes the interset between $\mathrm{S}_{0}$ and $\mathrm{S}_{1}$, when the distance is larger than $\omega_{1}$, the edge intensity is large enough. $D_{f}$ describes the intraset distance, if the edge intensity is small $D_{f}$ shows to be large. For an 8-bit gray level image, $\mathrm{L}$ is equal to 256. $f(i)$ shows the relationship of interset and the intraset distances, and provides values $f(i)$ for estimating .
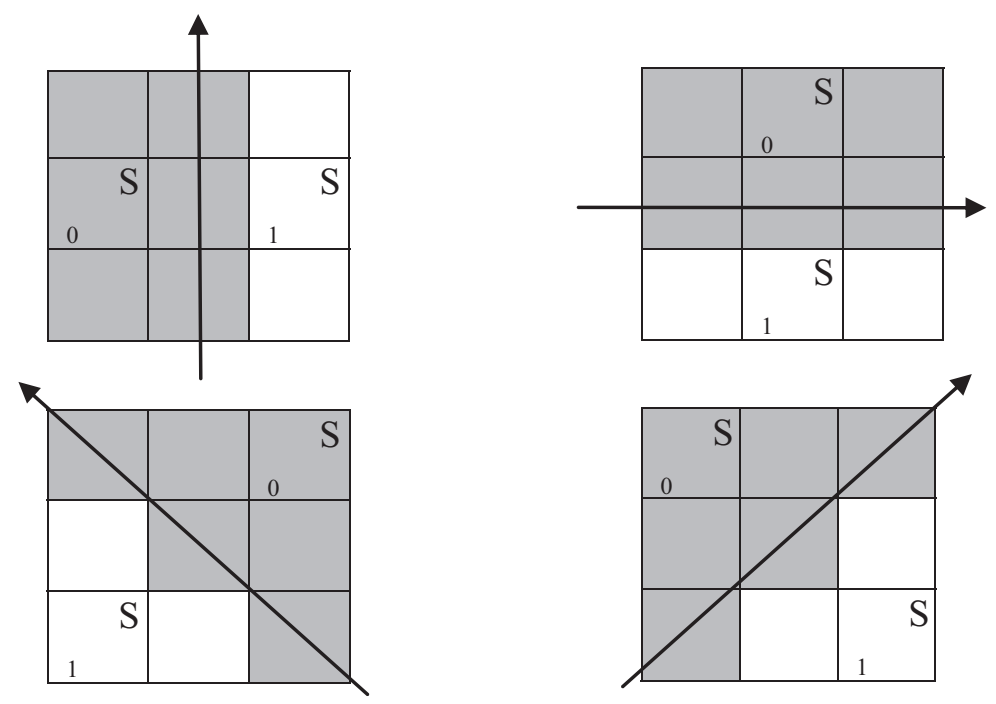

Fig. 1. Original 4 directions

Therefore, the edge points were located to differentiate the edge points and the noise point on the first step. For getting the exact location of the edge points, another 4 new added directions proposed by the improved method were showed in Fig.2. Hence, edge points and noise points were separated better, and the edge points can be located more accurately. 

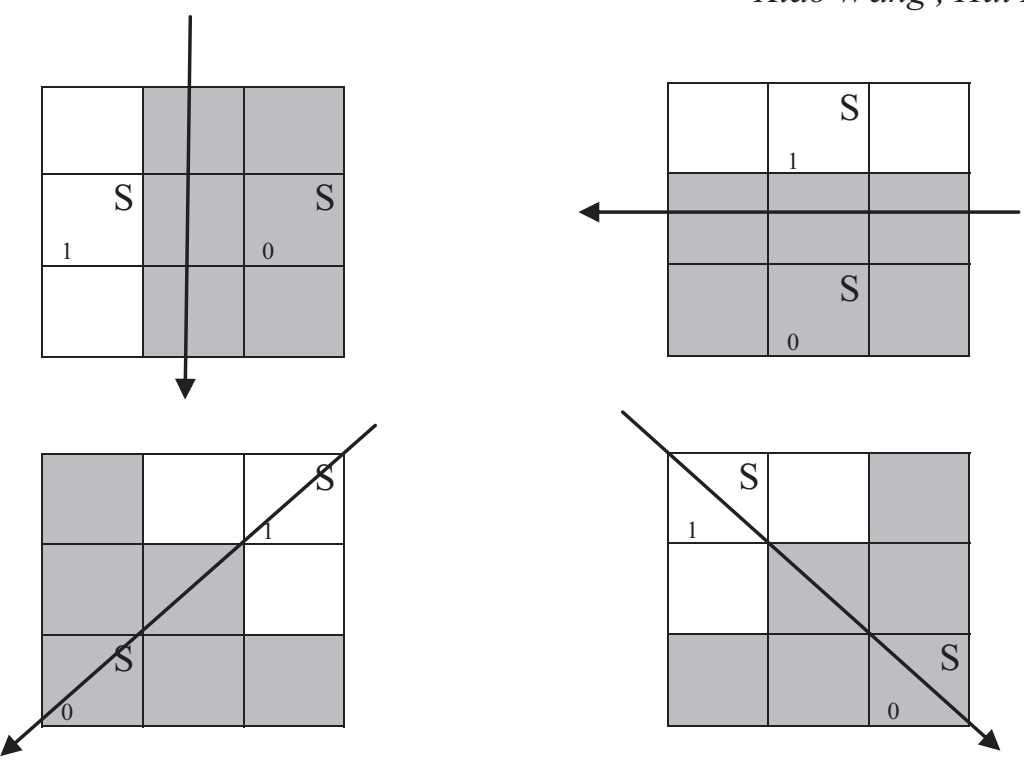

Fig. 2. The new 4 directions

From the function presented above, a better contour of an image was gotten without solving the noise problem. Since most of the edges were continuous with 'neighbors' but the noises were like single speckles, the gray-level continuity can be the judgment criterion. If the gray-level difference value between one point and its neighbor, which were got through the above function, was larger than a threshold $T$, this point mostly is a single speckle and should be considered as a noise point. Otherwise, the point was taken as it belongs to the edge domain. The principle was showed as follows:

$$
\begin{aligned}
& |e(i, j)-e(i-1, j-1)|<T,|e(i, j)-e(i+1, j+1)|<T \\
& |e(i, j)-e(i-1, j+1)|<T,|e(i, j)-e(i+1, j-1)|<T \\
& |e(i, j)-e(i-1, j)|<T,|e(i, j)-e(i+1, j)|<T \\
& |e(i, j)-e(i, j-1)|<T,|e(i, j)-e(i, j+1)|<T
\end{aligned}
$$

Where $e(i, j)$ was a pixel in a picture, $e(i-1, j-1), e(i+1, j+1)$ etc. were the neighbors of $e(i, j)$, and $T$ was the threshold.

If a pixel $e(i, j)$ had one of the characteristic showed in (4), the proposed method took it as an edge point, otherwise it should be taken as a noise point and eliminate it. The threshold $T$ was settled as 1.8 times of the gray-level average value, which was selected from plenty of experiments. Then, the job of edge detection and the noise problem both were completed. 
An Improved Edge Detection Method for Image Corrupted by

Gaussian Noise

\section{RESULT AND DISCUSSION}

A sample picture was used to compare the classical edge detection operators, the original algorithm and the references methods with the proposed method in Fig.3.

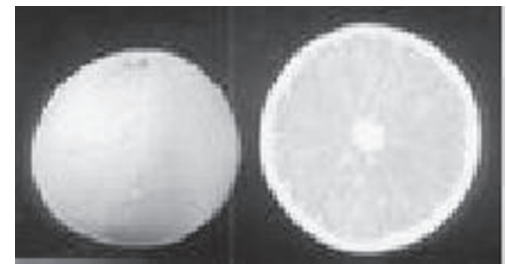

(a)

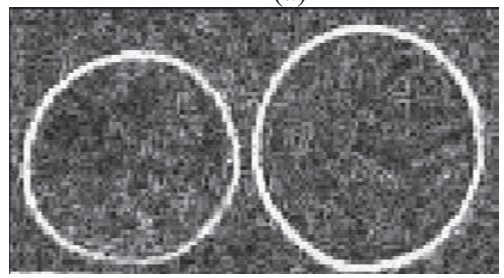

(c)

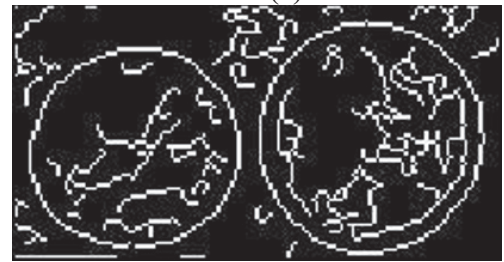

(e)

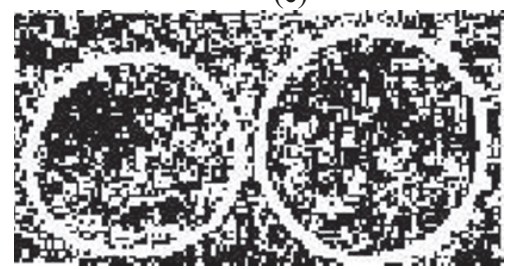

(g)

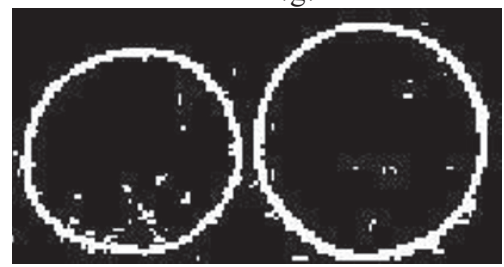

(i)

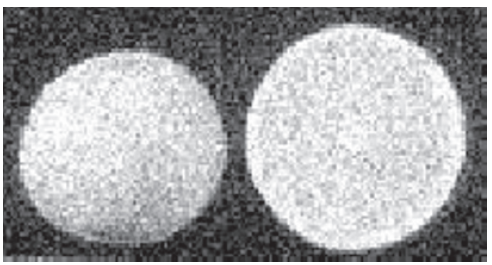

(b)

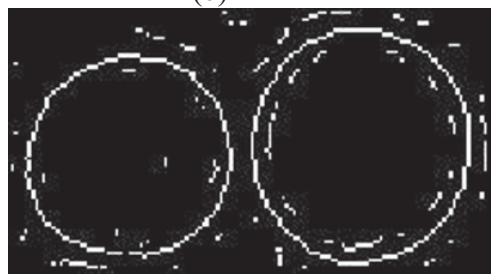

(d)

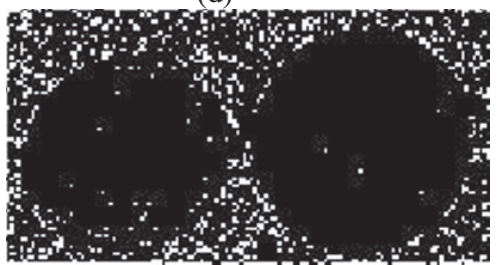

(f)

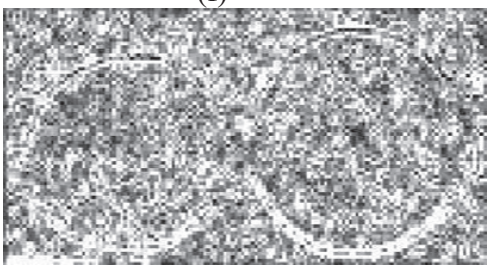

(h)

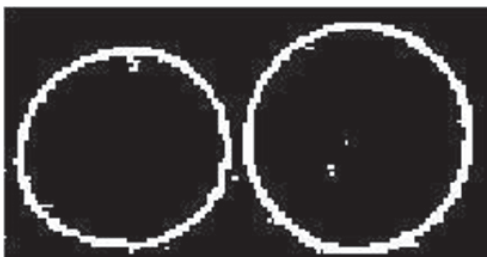

(j)

Fig.3. Comparisons with different algorithms for 'cameraman' image:

(a) the original 'cameraman' image; (b) original image added $2 \%$ gaussian noise; (c) the edge map by using original algorithm (Kang et al., 2007); (d) the result of LOG operator; (e) 
the result of Canny operator; (f) the result of the method base on gradient(Zhao et al., 2005) (g) the result of half neighborhood algorithm (Xu et al., 2006) (h) the result of the improved Sobel operator (Yao et al., 2007) (i) the result of original method with the denoising process; $(\mathrm{j})$ the edge map by using the proposed improved method with 8 directions and the denoising process.

According to the images in Figure 3, the results of classical edge detection operators (d), (e) in Figure 3 were confused by noise and edge, lots of noise points were considered as edge points and left in the results, they were full of speckles caused by the $2 \%$ Gaussian noise; the edge points and the noise points cannot be located respectively, the detected edge were not clear and definitude enough. The principle of the method based on the gradients (Zhao et al., 2005) was similar with the classical methods, better result was given without noise, but with $2 \%$ Gaussian noise, the map showed in (f) was full of speckles. (g) was gotten by the half neighborhood algorithm (Xu et al., 2006), 8 masks were used and the location of the edge points were decided by the pivotal threshold, the contour hardly can be seen in the result. A blurry image was seen in map (h), it was the improved Sobel method (Yao et al., 2007)which was added another 2 masks, because of the similar principle with classical methods and the gradient methods, the result showed serious sensitivity to the $2 \%$ Gaussian noise.

The original algorithm (Kang et al., 2007) showed in (c) was sensitive to noise, the detected edge was blurred and the whole map was a little dark for image analyzing. In comparison, the last two images (i) and (j) showed better edge responses, in the situation of 4 directions in (i), the bottom part still had some speckles caused by the Gaussian noise, it had apparent advantages than the prior methods, but it cannot eliminate the speckles successfully. Clearly the last one showed in (j) is more legible, continuous, and less infected by noise. The speckles seen in (i) were mostly eliminated, and the edge was maintained well and hardly affected. Hence, the plumpness of the fruit can be more clearly seen in the result map, and the proposed method obtains the best edge detection result among those edge detection algorithms.

\section{CONCLUSION}

In order to improve the edge points' location and solve the noise problem, an improved method based on the maximizing objecting function was proposed in this paper. Through adding 4 more directions, analyzing the basic difference between edge points and noise points and finding out a proper threshold by experiments, the edge points and eliminate noise points were relocated from the edge image. The simulation results had shown that the proposed improved method provides a better edge detection results with 
exact location and responses to edge points, it clearly showed the shape and the plumpness of the main objects in the picture. And the capacity to eliminate the noise makes the proposed method can be used in agricultural recognitions and some more practical image edge detection situations.

\section{REFERENCES}

Chung-Chia Kang, Wen-June Wang, A novel edge detection method based on the maximizing objective function, Pattern Recognition 40(2007), 609-618

DUAN Rui-ling, LI Qing-xiang, LI Yu-he, Summary of image edge detection, Optical Technique, Vol.3 No.3, May 2005, 415-419

XU Wei-dian, XU LI-chun, HU Yue-li, Adaptive edge detection using a half neighborhood algorithm, Journal of Shanghai University (Natural Science), Vol.12 No.2, April. 2006, 146-149

YAO Xing-zhong, HU Han-ping, LU Tong-wei, An improved Sobel operator method based on cat visual cortex, Computer Engineering and Applications, 2007,43(31), 64-67,70

ZHAO Chun-jiang, SHI Wen-kang, DENG Yong, Novel edge detection method based on gradient, Opto-Electronic Engineering, Vol.32 No.4, April. 2005, 86-88 